\title{
Control of Magnetotactic Bacterium in a Micro-Fabricated Maze
}

\author{
Islam S. M. Khali*, Marc P. Pichel*, Bart A. Reefman*, Ozlem Sardan Sukas ${ }^{\dagger}$, Leon Abelmann ${ }^{\dagger}$ \\ and Sarthak Misra* \\ University of Twente, The Netherlands
}

\begin{abstract}
We demonstrate the closed-loop control of a magnetotactic bacterium (MTB), i.e., Magnetospirillum magnetotacticum, within a micro-fabricated maze using a magneticbased manipulation system. The effect of the channel wall on the motion of the MTB is experimentally analyzed. This analysis is done by comparing the characteristics of the transient- and steady-states of the controlled MTB inside and outside a microfabricated maze. In this analysis, the magnetic dipole moment of our MTB is characterized using a motile technique (the u-turn technique), then used in the realization of a closed-loop control system. This control system allows the MTB to reach reference positions within a micro-fabricated maze with a channel width of $10 \mu \mathrm{m}$, at a velocity of $8 \mu \mathrm{m} / \mathrm{s}$. Further, the control system positions the MTB within a region-of-convergence of $10 \mu \mathrm{m}$ in diameter. Due to the effect of the channel wall, we observe that the velocity and the positioning accuracy of the MTB are decreased and increased by $71 \%$ and $44 \%$, respectively.
\end{abstract}

\section{INTRODUCTION}

The size of biological microrobots provides them with a wide range of applications, as opposed to miniature robots which usually fall in the range of a few millimeters to a few centimeters scale [1], [2]. These biological microrobots have the potential to perform targeted drug delivery and actuation of micro-objects [3], [4]. The natural propulsion mechanism allows for their navigation in low Reynolds number environments. Magnetotactic bacteria for instance, rotate their helical flagella to provide thrust force to overcome drag forces, while Tetrahymena pyriformis and Serratia marcescens use their cilia to provide self-propulsion [5], [6].

In order to predict how these biological microrobots will behave in vivo, key issues such as fluid flow velocity and the dynamics of the biological microrobots inside blood vessels have to be addressed. These issues can be investigated by analyzing the motion of the biological microrobots in microchannels. Martel et al. demonstrated the open-loop control of a swarm of MC-1 bacteria inside micro-channels of $50 \mu \mathrm{m}$ to $120 \mu \mathrm{m}$ in diameter [7], [8]. Open- and closed-loop control of a single magnetotactic bacteria, i.e., Magnetospirillum magnetotacticum (MS-1), were accomplished inside a flat capillary tube with an inner thickness of $200 \mu \mathrm{m}$ by Khalil et al. [9], [10].

This work addresses the closed-loop control of a magnetotactic bacterium (MTB) inside a micro-fabricated maze with channel width of $10 \mu \mathrm{m}$, shown in Fig. 1. Closed-loop control

\footnotetext{
*Islam S. M. Khalil, Marc P. Pichel, Bart A. Reefman, and Sarthak Misra are with MIRA-Institute for Biomedical Technology and Technical Medicine.

${ }^{\dagger}$ Ozlem Sardan Sukas and Leon Abelmann are with MESA+ Institute for Nanotechnology.
}

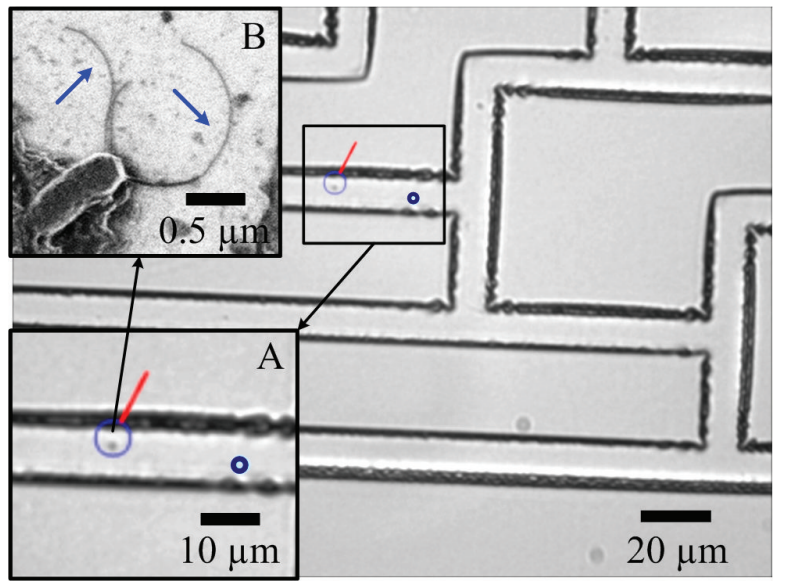

Fig. 1. Closed-loop control of a magnetotactic bacterium (MTB) inside a micro-fabricated maze. Point-to-point control of the MTB, i.e., Magnetospirillum magnetotacticum (MS-1), is accomplished under the influence of the applied magnetic fields. Inset A shows a controlled MTB moving towards a reference position. This motion control experiment is done to analyze the channel wall effect on the motion characteristics of the controlled MTB. The large blue circle indicates the controlled MTB, whereas the small blue circle indicates the reference position. The red line represents the velocity vector of the MTB. Inset A shows a Transmission Electron Microscope image of the propulsion mechanism (flagella) of the MTB, shown by the blue arrows

system is developed to accomplish point-to-point positioning of the MTB. This control capitalizes on the characterization of the magnetic dipole moment of the MTB using the $u$-turn technique [11], and is based on the magnetic force-current map of our magnetic system. The experimental results are done using our magnetic system, shown in Fig. 2 [12], [13]. We analyze the channel wall effect by comparing the motion control results inside and outside a micro-fabricated maze. Characteristics of the transient- and steady-states are used to evaluate the control system for each case.

The remainder of this paper is organized as follows: In Section II we discuss the theoretical background pertaining to the modeling and characterization of our MTB. First, the magnetic and drag forces and torques experienced by an MTB are modeled. Second, the u-turn technique is implemented to characterize the magnetic dipole moment of the MTB. In Section III, the characterized magnetic dipole moment is used in the realization of the magnetic forcecurrent map of our system. This map is used as a basis of our closed-control system. Section IV describes our experimental setup and provides motion control results. Finally, Section V concludes and provides directions for future work. 


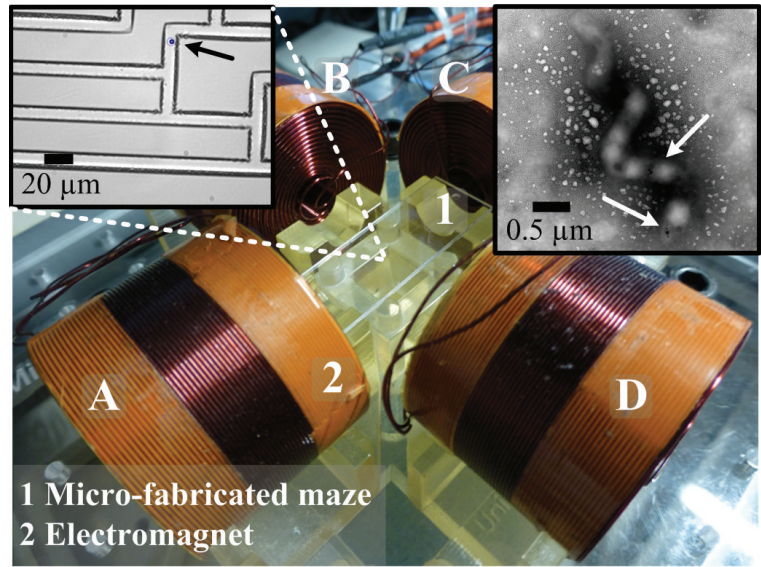

Fig. 2. Magnetic-based manipulation system for the wireless control of a magnetotactic bacterium (MTB) [12]. This magnetic system consists of an array of orthogonally oriented air-core electromagnets. The array surrounds a holder for a capillary tube (not shown) and a micro-fabricated maze. The capillary tube (VitroCom, VitroTubes 3520-050, Mountain Lakes, USA) is utilized in the characterization of the magnetic dipole moment using the $u$-turn technique and the control of the MTB in the absence of the channe wall effect, whereas the maze is used in the motion control experiments of the MTB to analyze the effect of the channel wall. The upper left inset shows the channels of the maze and the black arrow indicates the position of the MTB. The upper right inset shows a Transmission Electron Microscope image of the spiral membrane of the MTB, and the white arrows indicate positions of the magnetite nano-crystals

\section{Modeling And Characterization}

In this section, the magnetic and drag forces and torques experienced by an MTB are modeled. In addition, the magnetic dipole moment of the MTB is characterized using the u-turn technique [11].

\section{A. Modeling of Magnetotactic Bacterium}

Under the influence of a magnetic field, the magnetic force $\left(\mathbf{F}(\mathbf{P}) \in \mathbb{R}^{3 \times 1}\right)$ and torque $\left(\mathbf{T}(\mathbf{P}) \in \mathbb{R}^{3 \times 1}\right)$ experienced by an MTB located at position $\left(\mathbf{P} \in \mathbb{R}^{3 \times 1}\right)$ are given by

$$
\mathbf{F}(\mathbf{P})=(\mathbf{m} \cdot \nabla) \mathbf{B}(\mathbf{P}) \text { and } \mathbf{T}(\mathbf{P})=\mathbf{m} \times \mathbf{B}(\mathbf{P}),
$$

where $\mathbf{m} \in \mathbb{R}^{3 \times 1}$ and $\mathbf{B}(\mathbf{P}) \in \mathbb{R}^{3 \times 1}$ are the magnetic dipole moment of the MTB and the induced magnetic field, respectively. The magnetic torque, magnetic force and propulsion force should overcome the drag force $\left(F_{\mathrm{d}}\right)$ and torque $\left(T_{\mathrm{d}}\right)$

$$
F_{\mathrm{d}}=\gamma|\dot{\mathbf{P}}| \text { and } T_{\mathrm{d}}=\alpha \omega \text {. }
$$

In (2), $|\dot{\mathbf{P}}|$ and $\omega$ are the linear and angular velocities of the MTB, respectively. Further, $\gamma$ is the linear drag coefficient and is given by [14]

$$
\gamma=2 \pi \eta L\left[\ln \left(\frac{2 L}{d}\right)-0.5\right]^{-1},
$$

where $\eta, L$ and $d$ are the dynamic viscosity of the growth medium of the MTB, length and diameter of the MTB, respectively. Further, in (2), $\alpha$ is the rotational drag coefficient and is given by [15]

$$
\alpha=\frac{\pi \eta L^{3}}{3}\left[\ln \left(\frac{L}{d}\right)+0.92\left(\frac{d}{L}\right)-0.662\right]^{-1} .
$$

During the wireless control of an MTB, magnetic-based manipulation systems are utilized [9]. We consider a magnetic system with $n$-electromagnets. The magnetic field can be determined by the superposition of the contribution of each of the electromagnets [16]

$$
\mathbf{B}(\mathbf{P})=\sum_{i=1}^{n} \mathbf{B}_{i}(\mathbf{P})=\sum_{i=1}^{n} \widetilde{\mathbf{B}}_{i}(\mathbf{P}) I_{i}=\widetilde{\mathbf{B}}(\mathbf{P}) \mathbf{I} .
$$

where $\mathbf{B}_{i}(\mathbf{P})$ is the induced magnetic field by the $i$ th electromagnet. The magnetic field $\left(\mathbf{B}_{i}(\mathbf{P})\right)$ is linearly proportional to the current $\left(I_{i}\right)$ of the $i$ th electromagnet, as we use aircore electromagnets. Further, $\widetilde{\mathbf{B}}(\mathbf{P}) \in \mathbb{R}^{3 \times n}$ is a matrix which depends on the position at which the magnetic field is evaluated, and $\mathbf{I} \in \mathbb{R}^{n \times 1}$ is a vector of the applied current. The magnetic field due to each electromagnet is related to the current input by $\widetilde{\mathbf{B}}_{i}(\mathbf{P})$. Substituting (5) in the magnetic force equation (1) yields

$$
\mathbf{F}(\mathbf{P})=(\mathbf{m} \cdot \nabla) \widetilde{\mathbf{B}}(\mathbf{P}) \mathbf{I}=\boldsymbol{\Lambda}(\mathbf{m}, \mathbf{P}) \mathbf{I},
$$

where $\boldsymbol{\Lambda}(\mathbf{m}, \mathbf{P}) \in \mathbb{R}^{3 \times n}$ is the actuation matrix which maps the input currents onto magnetic forces. This actuation matrix depends on the magnetic dipole moment of the MTB and its position. Realization of this map necessitates the characterization of the magnetic dipole moment, and the evaluation of the magnetic field gradients at the position of the MTB.

A finite element (FE) model is developed for our magnetic-based manipulation system. Gradients of the magnetic fields are calculated within the workspace of the MTB, as shown in Fig. 3. We verify the accuracy of our FE model by measuring the magnetic fields using a calibrated threeaxis Hall magnetometer (Sentron AG, Digital Teslameter 3MS1-A2D3-2-2T, Switzerland) at 12 points that span the workspace of our magnetic system. The average deviation in the magnitude and direction of the magnetic field are $2.3 \%$ and $0.7 \%$, respectively. We apply 16 sets of current vectors and calculate the corresponding gradients. These sets are devised based on the saturation limits of our current amplifiers, i.e., Elmo 'Whistle' 1/60 servo controller (Elmo Motion Control, Petach-Tikva, Israel). Fig. 3 provides the field gradients for 4 different representative sets of current vectors, indicating constant gradients within the workspace of our magnetic system. This observation simplifies the implementation of our closed-loop control system since the pseudoinverse of the actuation matrix (6) is realized to calculate the current vector $(\mathbf{I})$.

\section{B. Characterization of the Magnetic Dipole Moment}

Realization of the force-current map (6), necessitates the characterization of the magnetic dipole moment of our MTB. Under magnetic field reversals, an MTB undergoes u-turn trajectories. The diameter $(D)$ of the $u$-turn is given by [11]

$$
D=\frac{\alpha \pi|\dot{\mathbf{P}}|}{|\mathbf{m} \| \mathbf{B}(\mathbf{P})|},
$$




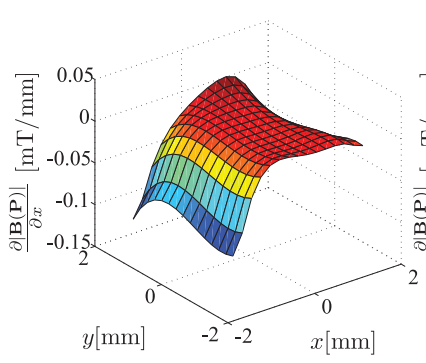

(a) Gradient along $x$-axis

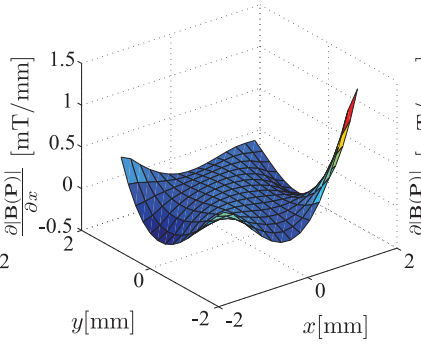

(b) Gradient along $x$-axis

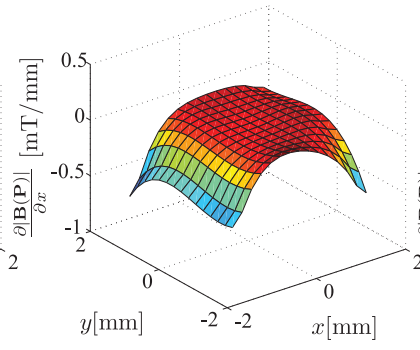

(c) Gradient along $x$-axis

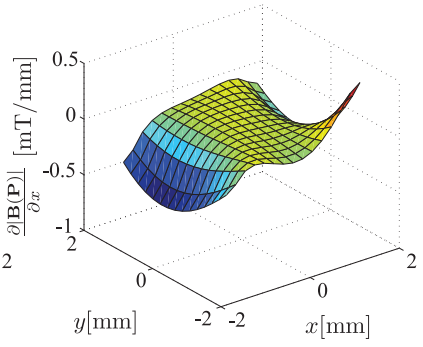

(d) Gradient along $x$-axis

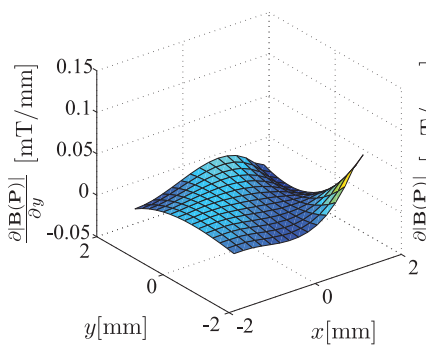

(e) Gradient along $y$-axis

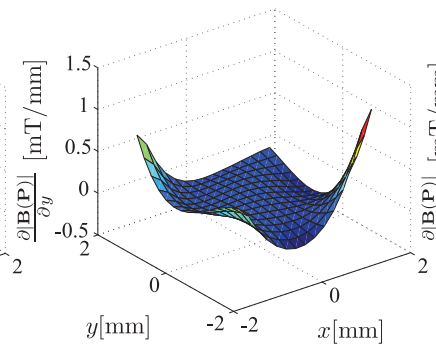

(f) Gradient along $y$-axis

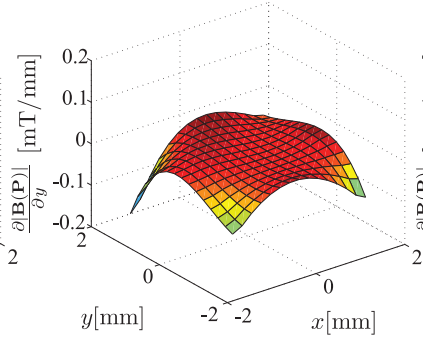

(g) Gradient along $y$-axis

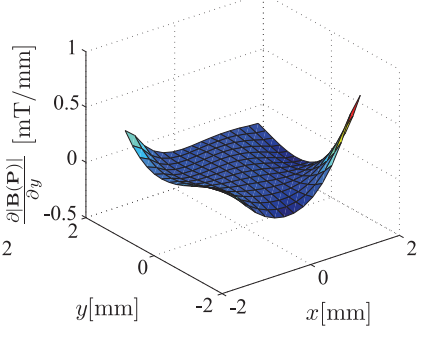

(h) Gradient along $y$-axis

Fig. 3. Results of the finite element (FE) analysis of our magnetic-based manipulation system. This system consists of four orthogonally-oriented air-core electromagnets. The FE analysis describes the magnetic field gradients within a workspace of $2 \times 2 \mathrm{~mm}^{2}$ when the following representative current

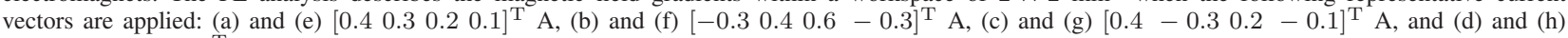
$\left[\begin{array}{lll}0.5 & 0.3 & 0.8-0.1\end{array}\right]^{\mathrm{T}}$ A. The entries of the aforementioned current vectors are applied to electromagnets A, B, C and D, respectively. These results are utilized in the realization of the force-current map (6) and its inverse. The gradients of the magnetic field are almost constant within the center of the workspace of our system. This observation simplifies the implementation of the control system since the gradients do not have to be calculated at each point of the workspace. Our FE model accounts for the couplings between the electromagnets. Further, the deviation in magnitude and angle between our FE model and measurements are $2.3 \%$ and $0.7 \%$, respectively. The FE model is created using Comsol Multiphysics ${ }^{\circledR}$ (COMSOL, Inc., Burlington, U.S.A).

whereas, the $u$-turn time $(\tau)$ is given by

$$
\tau=\frac{\alpha}{|\mathbf{m} \| \mathbf{B}(\mathbf{P})|} \ln \left(\frac{2|\mathbf{m} \| \mathbf{B}(\mathbf{P})|}{\mathrm{k} T}\right),
$$

where $\mathrm{k}$ and $T$ are the Boltzmann constant and the temperature of the fluid, respectively. Characterization of the magnetic dipole is carried out using our magnetic system, shown in Fig. 2. A culture of magnetotactic bacteria in $0.02 \mathrm{ml}$ of growth medium are incubated within a capillary tube (VitroCom, VitroTubes 3520-050, Mountain Lakes, USA). This tube has an inner-width and inner-thickness of $1 \mathrm{~mm}$ and $0.2 \mathrm{~mm}$, respectively. The bacterial density ranges from $10^{6} / \mathrm{ml}$ to $10^{7} / \mathrm{ml}$. The Magnetospirillum magnetotacticum (MS-1) cultures utilized in our work are grown according to the protocol provided by Bertani et al. [17]. Electromagnets $\mathrm{A}$ and $\mathrm{C}$ (Fig. 2) are utilized to provide uniform magnetic fields, then the magnetic field is reversed. The diameter and time of the $u$-turn trajectory is determined from the off-line motion analysis of the MTB. Fig. 4 shows the u-turn trajectory taken by the MTB during the reversal of the magnetic field. Using (7) and (8), the average magnetic dipole moment is determined from 15 different $u$-turn trajectories. The magnetic dipole moment of our MTB has an average of $1.6 \times 10^{-16}$ A.m ${ }^{2}$, at magnetic field of $7.9 \mathrm{mT}$, and linear velocity of $32 \mu \mathrm{m} / \mathrm{s}$. In the calculation of the magnetic dipole moment using (7) and (8), the rotational drag coefficient $(\alpha)$ is computed using (4) based on the morphology of the MTB and the properties of the growth medium [9], [10]. We assume that the growth medium has similar properties as water.

\section{Closed-LOOP CONTROL}

Closed-loop control of an MTB is accomplished by directing the field lines towards a reference position, then the MTB performs a flagellated swim towards this reference position. Due to the self-propulsion force provided by the rotation of the helical flagella, the closed-loop control system can only locate the MTB within the vicinity of the reference position.

\section{A. Control System Design}

In a low Reynolds number environment (inertial terms are ignored), motion of an MTB is governed by

$$
|\mathbf{F}(\mathbf{P})|+F_{\mathrm{d}}+f=0 \text { and }|\mathbf{T}(\mathbf{P})|+T_{\mathrm{d}}+\Omega=0,
$$

where $f$ and $\Omega$ are the force and torque generated by each helical flagella, respectively. We use the force equation in (9), to generate the desired currents at each of the electromagnets. In order to realize the closed-loop control system, we calculate the position and velocity tracking errors as follows:

$$
\mathbf{e}=\mathbf{P}-\mathbf{P}_{\text {ref }} \text { and } \dot{\mathbf{e}}=\dot{\mathbf{P}}-\dot{\mathbf{P}}_{\text {ref }}=\dot{\mathbf{P}} \text {. }
$$

In (10), e and $\dot{e}$ are the position and velocity tracking errors, respectively. Further, $\mathbf{P}_{\text {ref }}$ is a fixed reference position. We devise a controlled magnetic force $\left(\mathbf{F}_{\text {des }}(\mathbf{P})\right)$ of the form

$$
\mathbf{F}_{\text {des }}(\mathbf{P})=\mathbf{K}_{\mathbf{p}} \mathbf{e}+\mathbf{K}_{\mathbf{d}} \dot{\mathbf{e}}
$$




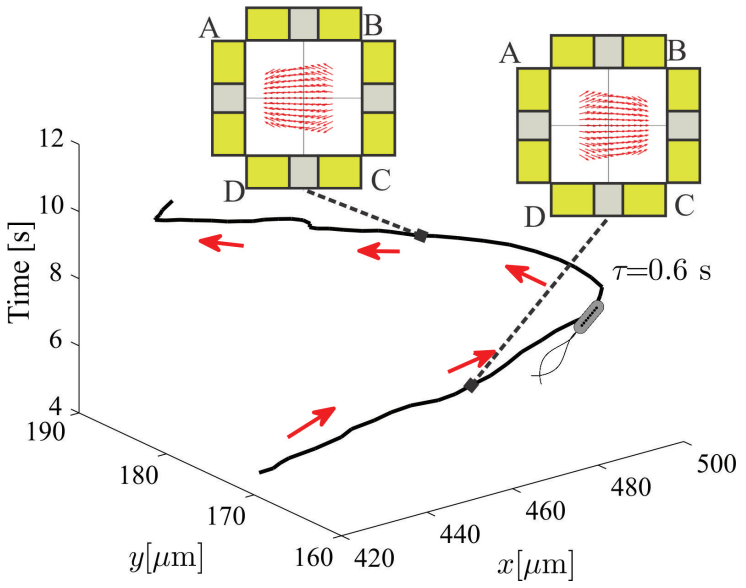

(a) MTB undergoes a $u$-turn trajectory

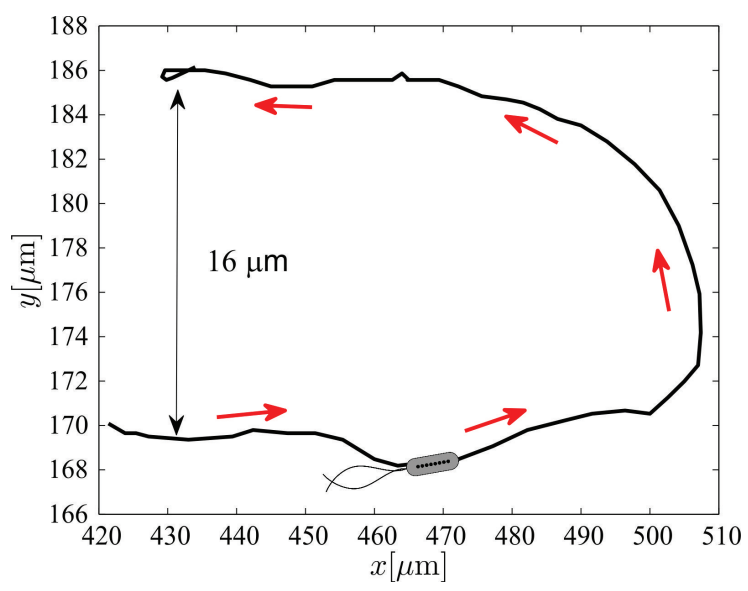

(b) Diameter of the u-turn trajectory

Fig. 4. Characterization of the magnetic dipole moment of a magnetotactic bacterium (MTB) using the $u$-turn technique [11]. (a) Motion of the MTB before and after a field reversal. The MTB (length $\sim 5 \mu \mathrm{m}$ ) undergoes a $u$-turn trajectory, during the field reversal. The red arrows indicate the direction of the MTB. (b) The average u-turn diameter $(\sim 16 \mu \mathrm{m})$ and time $(\tau=0.6 \mathrm{~s})$ are used to estimate the magnetic dipole moment. The $u$-turn time is determined starting from the initiation time of the field reversal until the MTB aligns itself along the field lines. The average magnetic dipole moment for 15 magnetotactic bacteria is $1.6 \times 10^{-16} \mathrm{~A} . \mathrm{m}^{2}$ at a magnetic field of $7.9 \mathrm{mT}$, and a linear velocity of $32 \mu \mathrm{m} / \mathrm{s}$. Magnetic dipole moment is calculated using

(7) and (8). Please refer to the attached video that demonstrates the u-turn technique utilized for the characterization of the magnetic dipole moment.

where $\mathbf{K}_{\mathbf{p}}$ and $\mathbf{K}_{\mathbf{d}}$ are the controller positive-definite gain matrices and are given by

$$
\mathbf{K}_{\mathbf{p}}=\left[\begin{array}{cc}
k_{\mathrm{p} 1} & 0 \\
0 & k_{\mathrm{p} 2}
\end{array}\right] \text { and } \mathbf{K}_{\mathbf{d}}=\left[\begin{array}{cc}
k_{\mathrm{d} 1} & 0 \\
0 & k_{\mathrm{d} 2}
\end{array}\right]
$$

In (12), $k_{\mathrm{p} i}$ and $k_{\mathrm{d} i}$, for $(i=1,2)$, are the proportional and derivative gains, respectively. Substitution of (11) in (9), i.e., $\mathbf{F}_{\text {des }}(\mathbf{P})=\mathbf{F}(\mathbf{P})$, and assuming zero propulsion force $(f=$ $0)$ yields the following position tracking error dynamics:

$$
\dot{\mathbf{e}}+\left(\mathbf{K}_{\mathbf{d}}+\gamma \mathbf{\Pi}\right)^{-1} \mathbf{K}_{\mathbf{p}} \mathbf{e}=0,
$$

where $\Pi \in \mathbb{R}^{2 \times 2}$ is the identity matrix. We only consider motion control of the MTB in a two-dimensional workspace, and based on (13), the controller gains must be selected such that the matrix $\left(\mathbf{K}_{\mathbf{d}}+\gamma \mathbf{\Pi}\right)^{-1} \mathbf{K}_{\mathbf{p}}$ is positive definite.

\section{B. Region-of-Convergence}

Since motile MTB provides propulsion by its helical flagella, $f \neq 0$. Therefore, the closed-loop control system does not allow the position tracking error to go to zero, it rather locates the MTB within the vicinity of the reference position, i.e., region-of-convergence. Positioning accuracy of the control system can be evaluated by the diameter of the region-of-convergence. From (13), the size of the region-ofconvergence depends on the gains of the control system, the propulsion force of the flagella and the dynamic viscosity of the growth medium.

\section{EXPERIMENTAL RESULTS}

Our motion control experiments are done using a magnetic-based manipulation system with four orthogonallyoriented electromagnets (Fig. 2). Position of the MTB is determined by a microscopic system and a feature tracking software. This software is based on subtracting the consecutive images obtained by our microscopic system to suppress the static edges of the channels, then motion of the MTB is tracked by computing the image gradients within a window (shown by the blue circle in inset B of Fig. 1) around the MTB. Detailed explanations of this magnetic system are provided in [12], [13]. The array of electromagnets surrounds a capillary tube or a micro-fabricated maze. Experiments are conducted using the capillary tubes to analyze the performance of the control system in the absence of the channel wall effect, whereas the micro-fabricated channel provides this effect for the control system.

\section{A. Micro-Fabricated Maze}

Our micro-fabricated maze is made of glass owing to its mechanical robustness and optical transparency. Procedures of fabrication are provided in Fig. 5. First, a glass (Borofloat) wafer is cleaned using nitric acid solutions. The thickness and diameter of this wafer are $500 \mu \mathrm{m}$ and $100 \mathrm{~mm}$, respectively (Fig. 5(a)). The layout of our micro-fabricated maze is transferred on the top-side of the wafer by spin- (a)

(c)

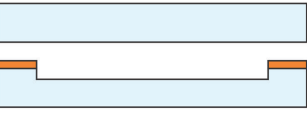

(e)

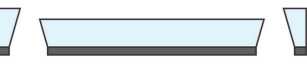

(b)

(d)

(f)

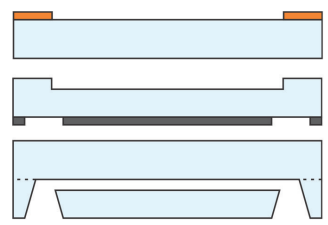

$\square$ Glass $\square$ Photoresist (Olin OiR 908-35) $\square$ Photoresist (Ordyl Bf410)
Fig. 5. Fabrication steps of the micro-fabricated maze: (a) Glass wafer (b) Definition of the layout of the maze using ultraviolet (UV) lithography. (c) Etching of the maze by deep reactive-ion etching. (d) Development of the reservoirs using UV lithography. (e) Powder blasting of the inlet and outlets. (f) Thermal bonding of two glass wafers. 


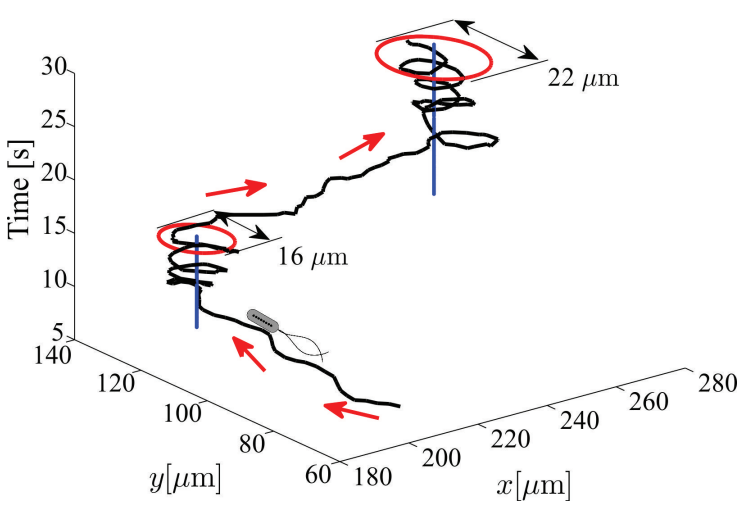

(a) Closed-loop control of an MTB

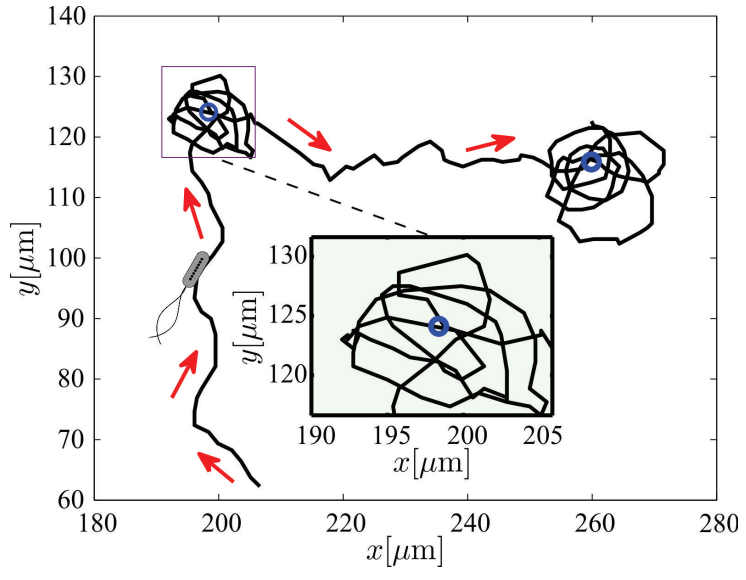

(b) Path of the controlled MTB

Fig. 6. Representative closed-loop control of a magnetotactic bacterium (MTB) inside a capillary tube with inner-width and -thickness of 1 mm and $0.2 \mathrm{~mm}$, respectively. The MTB is controlled using control law (11). The controller gains are: $k_{\mathrm{p} 1}=k_{\mathrm{p} 2}=15.0 \mathrm{~s}-2$ and $k_{\mathrm{d} 1}=k_{\mathrm{d} 2}=15.5 \mathrm{~s}-1$. The red arrows indicate the direction of the controlled MTB. (a) An MTB follows two reference positions at a velocity of $24 \mu \mathrm{m} / \mathrm{s}$. The solid blue lines represent two reference positions. (b) Our closed-loop control system positions the MTB within the vicinity of the reference positions. The inset shows that the MTB is positioned within a region-of-convergence of $16 \mu \mathrm{m}$ in diameter. The blue circles represent the two reference positions. Please refer to the attached video that demonstrates the results of the closed-loop control of the MTB inside the capillary tube.

coating a $3.5 \mu \mathrm{m}$ photoresist layer (Olin OiR 908-35) and ultraviolet (UV) exposure (Fig. 5(b)). This photoresist layer is then used as a mask in the realization of microchannels of $5 \mu \mathrm{m}$ in depth, through a deep reactive-ion etching process (Fig. 5(c)). The bottom-side of the wafer is coated by a $100 \mu \mathrm{m}$ photoresist layer (Ordyl BF410). One inlet and six outlets with diameter of $1.7 \mathrm{~mm}$ are defined by UV-exposure (Fig. 5(d)), then developed by etching the glass wafer using powder blasting with $29 \mu \mathrm{m}$ alumina particles (Fig. 5(e)). The wafer is then washed with deionized water, immersed in acetone and isopropanol, and cleaned by nitric acid solutions. Finally, the wafer is thermally bonded to an unprocessed glass wafer (Fig. 5(f)), then each micro-fabricated maze is diced to fit into a chip holder (FC-FC4515 chip holder, Micronit Microfluidics, Enschede, The Netherlands).

\section{B. Control Outside the Micro-fabricated Maze}

In the absence of the channel wall effect, the control law (11) allows for the positioning of the MTB within the vicinity of two reference positions, shown by the representative motion control result in Fig. 6. In this experiment, the MTB is incubated in a capillary tube (Section II-B). We observe that the MTB follows the reference positions at an average velocity of $28 \mu \mathrm{m} / \mathrm{s}$. In addition, the closed-loop control system positions the MTB within an average region-ofconvergence of $18 \mu \mathrm{m}$ in diameter. The average is calculated from 10 motion control trials inside a capillary tube.

\section{Control Inside the Micro-fabricated Maze}

We control the MTB inside the micro-fabricated maze (Fig. 2), to analyze the channel wall effect on the velocity and the positioning accuracy of the controlled MTB. Fig. 7 provides the experimental result of the MTB inside the maze. Control system (11) allows the MTB to follow two reference positions indicated by the small blue circles. We observe that the MTB is positioned within the vicinity of the reference positions, and the region-of-convergence is $10 \mu \mathrm{m}$. The control system positions the MTB at a velocity of $8 \mu \mathrm{m} / \mathrm{s}$. Table I provides a comparison between the characteristics of the controlled MTB outside and inside the micro-fabricated maze. The transient- and steady-states are analyzed by the velocity of the MTB and the size of the region-of-convergence, respectively.

\section{CONCLUSIONS AND FUTURE WORK}

We investigate the closed-loop motion control of an MTB inside a micro-fabricated maze to analyze the effect of the channel wall. Motion control experiments are conducted outside and inside a maze with a channel width of $10 \mu \mathrm{m}$. We do not only observe a $71 \%$ decrease in the linear velocity of the MTB, inside the maze, but we are also able to obtain $44 \%$ higher positioning accuracy.

Future work in this field should focus on the investigation of the effect of variable flow rate of the fluid. This investigation should be done to predict whether the flagella and magnetic forces are capable of holding the MTB at a reference position against a fluid flow. In addition, a threedimensional (3D) magnetic system with auto-focusing is

TABLE I

CHARACTERISTICS OF THE MAGNETOTACTIC BACTERIUM IN THE TRANSIENT- AND STEADY-STATES. CASE I: ClOSED-LOOP CONTROL OUTSIDE THE MICRO-FABRICATED MAZE (INSIDE A CAPILLARY TUBE). CASE II: ClosED-LOOP CONTROL INSIDE THE MAZE.

\begin{tabular}{lcc}
\hline Characteristics & Case I & Case II \\
\hline Velocity (transient-state) $[\mu \mathrm{m} / \mathrm{s}]$ & 28 & 8 \\
Region-of-convergence (steady-state) $[\mu \mathrm{m}]$ & 18 & 10 \\
\hline
\end{tabular}




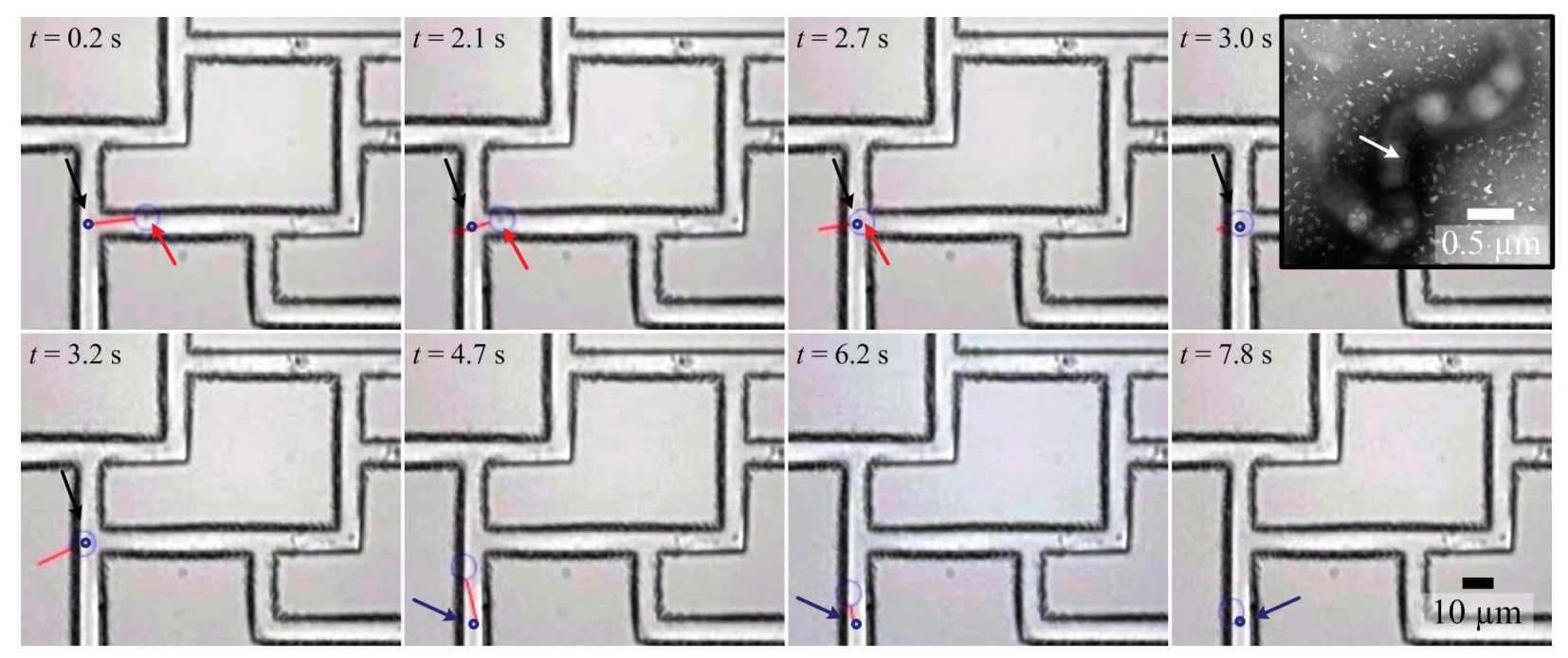

Fig. 7. Closed-loop control of a magnetotactic bacterium (MTB) inside a micro-fabricated maze with inner-width and -thickness of $10 \mu \mathrm{m}$ and $5 \mu \mathrm{m}$, respectively, at various time $(t)$ instants. The MTB is controlled using the control law (11). The controller gains are: $k_{\mathrm{p} 1}=k_{\mathrm{p} 2}=15.0 \mathrm{~s}-2$ and $k_{\mathrm{d} 1}=k_{\mathrm{d} 2}=15.5 \mathrm{~s}^{-1}$. This control system positions the MTB at a velocity of $8 \mu \mathrm{m} / \mathrm{s}$ and within a region-of-convergence of $10 \mu \mathrm{m}$. The black and blue arrows indicate the first and second reference positions, respectively. The small blue circles indicate these reference positions, whereas the large blue (light) circle is assigned by our feature tracking software [12]. The red (light) line represents the velocity vector of the MTB. The red arrows indicate the controlled MTB. The upper right inset shows a Transmission Electron Microscope image of an MTB, the white arrow indicates a chain of magnetite nano-crystals. Please refer to the attached video that demonstrates the results of the closed-loop control of the MTB inside the micro-fabricated maze.

essential to study the behavior and control the motion of the MTB in 3D space. Therefore, our magnetic system will be redesigned to allow for the visual tracking and control of the MTB in 3D space, and will be incorporated with a clinical imaging modality.

\section{REFERENCES}

[1] P. Valdastri, E. Sinibaldi, S. Caccavaro, G. Tortora, A. Menciassi, and P. Dario, "A Novel Magnetic Actuation System for Miniature Swimming Robots," IEEE Transactions on Robotics, vol. 27, no. 4, pp. 769-779, August 2011

[2] B. J. Nelson, I. K. Kaliakatsos, and J. J. Abbott, "Microrobots for minimally invasive medicine," The Annual Review of Biomedical Engineering, vol. 12, pp. 55-85, April 2010.

[3] S. Martel, O. Felfoul, J.-B. Mathieu, A. Chanu, S. Tamaz, M. Mohammadi, M. Mankiewicz, and N. Tabatabaei, "MRI-based medical nanorobotic platform for the control of magnetic nanoparticles and flagellated bacteria for target interventions in human capillaries," The International Journal of Robotics Research, vol. 28, no. 9, pp. 1169 1182, September 2009.

[4] S. Martel and M. Mohammadi, "Using a swarm of self-propelled natural microrobots in the form of flagellated bacteria to perform complex micro-assembly tasks," in Proceedings of The IEEE International Conference on Robotics and Automation, pp. 500-505, Alaska, USA, May 2010.

[5] D. H. Kim, P. S. S. Kim, A. A. Julius, and M. J. Kim, "Threedimensional control of engineered motile cellular microrobots," in Proceedings of the IEEE International Conference on Robotics and Automation (ICRA), pp. 721-726, Minnesota, USA, May 2012.

[6] M. S. Sakar, E. B. Steager, D. H. Kim, A. A. Julius, M. Kim, V. Kumar and G. J. Pappas, "Modeling, control and experimental characterization of microbiorobots," The International Journal of Robotics Research, vol. 30, no. 6, pp. 647-658, May 2011.

[7] Z. Lu and S. Martel, "Controlled bio-carriers based on magnetotactic bacteria," in Proceedings of The IEEE International Conference on Solid-State Sensors, Actuators and Microsystems, pp. 683-686, Lyon, France, June 2007.

[8] Z. Lu and S. Martel, "Preliminary investigation of bio-carriers using magnetotactic bacteria," in Proceedings of The IEEE Engineering in Medicine and Biology Society Annual International Conference (EMBS), pp. 683-686, New York City, USA, September 2006.

[9] I. S. M. Khalil, M. P. Pichel, L. Zondervan, L. Abelmann, and S. Misra, "Characterization and control of biological microrobots," in Proceedings of the 13th International Symposium on Experimental Robotics-Springer Tracts in Advanced Robotics, Quebec City, Canada, June 2012

[10] I. S. M. Khalil, M. P. Pichel, L. Abelmann, and S. Misra, "Closedloop control of magnetotactic bacteria," in The International Journal of Robotics Research. In Press

[11] A. S. Bahaj and P. A. B. James, "Characterisation of magnetotactic bacteria using image processing techniques," IEEE Transactions on Magnetics, vol. 29, no. 6, pp. 3358-3360, November 1993.

[12] J. D. Keuning, J. de Vries, L. Abelmann, and S. Misra, "Imagebased magnetic control of paramagnetic microparticles in water," in Proceedings of the IEEE International Conference of Robotics and Systems (IROS), pp. 421-426, San Francisco, USA, September 2011.

[13] I. S. M. Khalil, J. D. Keuning, L. Abelmann, and S. Misra, "Wireless magnetic-based control of paramagnetic microparticles," in Proceedings of the IEEE RAS/EMBS International Conference on Biomedical Robotics and Biomechatronics (BioRob), pp. 460-466, Rome, Italy, June 2012.

[14] H. C. Berg, "Random walks in biology," Princeton University Press, Princeton, New Jersey, 1993.

[15] Y. R. Chemla, H. L. Grossman, T. S. Lee, J. Clarke, M. Adamkiewicz, and B. B. Buchanan, "A new study of bacterial motion: superconducting quantum interference device microscopy of magnetotactic bacteria,” Biophysical Journal, vol. 76, pp. 3323-3330, June 1999.

[16] M. P. Kummer, J. J. Abbott, B. E. Kartochvil, R. Borer, A. Sengul, and B. J. Nelson, "OctoMag: an electromagnetic system for 5-DOF wireless micromanipulation," IEEE Transactions on Robotics, vol. 26, no. 6, pp. 1006-1017, December 2010.

[17] L. E. Bertani, J. Weko, K. V. Phillips, R. F. Gray, and J. L. Kirschvink, "Physical and genetic characterization of the genome of Magnetospirillum magnetotacticum, strain MS-1," International Journal on Genes and Genomes, vol. 264, pp. 257-263, January 2001.

[18] I. S. M. Khalil, R. M. P. Metz, L. Abelmann, and S. Misra, "Interaction force estimation during manipulation of microparticles," in Proceedings of the IEEE International Conference of Robotics and Systems (IROS), pp. 950-956, Vilamoura, Portugal, October 2012 